\title{
Review of: "Urinary actin, as a potential marker of sepsis-related acute kidney injury: A pilot study"
}

\author{
Catarina Cardoso ${ }^{1}$, Silvia Coelho \\ 1 Hospital Garcia de Orta
}

Potential competing interests: The author(s) declared that no potential competing interests exist.

This was a single center study conducted in a 16 bedded central ICU in Hungary. The main goal of the authors was to measure urinary actin (U-actin) in control and septic (with and without AKI) patients and describe its time course to test if it could be used as a predictor of acute kidney injury (AKI) and mortality.

We believe that are several methodology characteristics that should be taken into account when interpreting the data.

- First, the sample characteristics. The total of patients enrolled was small, which gives the study hardly any power to infer robust conclusions. An example of the higher probability of misguided results is the decreasing mortality rate between patients in AKI with increasing stages of severity $(80 \%, 71.4 \%$ and $52.6 \%$, in stage 1, 2 and 3 respectively). This contradicts the data available by several published multicenter prospective and retrospective cohort studies ${ }^{1,2}$, but the authors did not comment it. Furthermore, considering the inclusion time of the study (4 years), the size of the ICU (16 beds), the small number of exclusion criteria and the consecutive sampling described in the methods section, we would expect that a high number of patients could fulfil the inclusion criteria and be enrolled. In fact, authors acknowledge it was difficult to recruit consecutive patients, and that raises the possibility of not having a random sample and the creation of biased groups, that are not possible to compare. Demographic and baseline characteristics were different between sepsis and sepsis + AKI groups and results were not adjusted to variables with impact on renal and patient outcome, such as age. Also, the sample size (in sepsis and sepsis $+\mathrm{AKI}$ ) decreases with time and no explanation was given about that. Could it be that they were transferred, died, or started renal replacement therapy (RRT)? Moreover, the authors do not explain how controls were chosen.

- Second, in the study design, it would have been interesting to exclude all chronic kidney disease (CKD) patients (not only the ones with end-stage renal disease or kidney transplantation) or use them as an independent group, since they are more prone to AKI which can lead to confounding results. The evidence considering CKD as a risk factor for AKI is growing ${ }^{3}$. Having this thought in mind and even though the absolute numbers of CKD patients included in the study is not apparently significant, they account for more than $10 \%$ of the patients in sepsis and sepsis + AKI groups and none in the control group. Additionally, and as said in the discussion, the role of u-actin in CKD is not yet defined and other 
causes of CKD should be explored.

- Third, the authors could not aim to explore if u-actin could predict AKI as they proposed themselves because the AKI group already had established AKI at inclusion time. AKI is one of the most frequent organ dysfunctions associated with sepsis and septic shock. In the ICU it is considered an independent risk factor for death, associated with short-term mortality rates that exceed $50 \%$ and have only slightly declined in the last 50 years ${ }^{3}$. In the last decades, many serum and urinary biomarkers were discovered, aiming to help clinicians diagnose AKI earlier and improve prognosis. ${ }^{4}$ [TIMP-2]•[IGFBP7], a cell cycle arrest biomarker, is currently considered the most promising predictive biomarker of AKI and has already been validated in several clinical scenarios. ${ }^{5}$

- Forth, it would have been interesting to explore the patients' outcome (which they did, although not adjusting to important variables) and renal prognosis (that was not performed). More recently, research has shifted its focus from diagnosis to recovery, trying to predict the persistence of renal dysfunction and potential mechanisms of kidney tissue damage and repair in acute kidney injury. The RUBY study was the most promising research in this field to date, leading to the discovery of C-C motif chemokine ligand 14 (CCL14), a predictor of renal non-recovery after AKI. ${ }^{6}$

A strong point was the methodology created for determining u-actin that is reproducible for other studies.

\section{Bibliography:}

1. Jiang, L., et al "Epidemiology of acute kidney injury in intensive care units in Beijing: the multi-center BAKIT study", BMC Nephrol (2019) 20:468. https://doi.org/10.1186/s12882-019-1660-z

2. Mandelbaum, T., "Outcome of Critically ill Patients with Acute Kidney Injury using the AKIN Criteria", Crit Care Med. 2011 December; 39(12): 2659-2664. doi:10.1097/CCM.0b013e3182281f1b

3. Kellum, J.A., Romagnani, P., Ashuntantang, G. et al. "Acute kidney injury". Nat Rev Dis Primers 7, 52 (2021). https://doi.org/10.1038/s41572-021-00284-z

4. Oh, D-J, "A long journey for acute kidney injury biomarkers", RENAL FAILURE 2020, VOL. 42, NO. 1, 154165. https://doi.org/10.1080/0886022X.2020.1721300

5. Kashani, K., et al. “Discovery and validation of cell cycle arrest biomarkers in human acute kidney injury". Crit Care. 2013; 17 (1): R25

6. Hoste, E., et al. "Identifcation and validation of biomarkers of persistent acute kidney injury: the RUBY study". Intensive Care Med (2020) 46:943-953 https://doi.org/10.1007/s00134-019-05919-0 
